\title{
Frontières
}

\section{Autodétermination et résilience en réadaptation avec l'outil Priorités d'intervention du client (PIC)}

\author{
Eduardo Cisneros, Audrey-Kristel Barbeau, Gilles Charette, Geneviève Léveillé \\ et Michelle McKerral
}

Volume 22, numéro 1-2, automne-printemps 2009-2010

Résilience et deuil

URI : https://id.erudit.org/iderudit/045031ar

DOI : https://doi.org/10.7202/045031ar

Aller au sommaire du numéro

Éditeur(s)

Université du Québec à Montréal

ISSN

1180-3479 (imprimé)

1916-0976 (numérique)

Découvrir la revue

Citer cet article

Cisneros, E., Barbeau, A.-K., Charette, G., Léveillé, G. \& McKerral, M. (2009).

Autodétermination et résilience en réadaptation avec l'outil Priorités

d'intervention du client (PIC). Frontières, 22(1-2), 85-88.

https://doi.org/10.7202/045031ar
Résumé de l'article

Cet article présente la façon dont l'outil interdisciplinaire Priorités d'intervention du client (PIC) fait appel à la résilience et à l'autodétermination soutenant le client dans le choix de ses objectifs d'intervention. Le PIC a été développé en contexte de réadaptation pour des personnes ayant un traumatisme craniocérébral. Il s'agit toutefois d'un outil interdisciplinaire, non spécifique à une discipline, à une clientèle ou à un contexte clinique, qui permet à diverses clientèles de cibler leurs priorités d'intervention ainsi que leurs forces, favorisant ainsi l'actualisation de leurs capacités d'adaptation et de résilience. 


\section{Résumé}

Cet article présente la façon dont l'outil interdisciplinaire Priorités d'intervention du client (PIC) fait appel à la résilience et à l'autodétermination soutenant le client dans le choix de ses objectifs d'intervention. Le PIC a été développé en contexte de réadaptation pour des personnes ayant un traumatisme craniocérébral. II s'agit toutefois d'un outil interdisciplinaire, non spécifique à une discipline, à une clientèle ou à un contexte clinique, qui permet à diverses clientèles de cibler leurs priorités d'intervention ainsi que leurs forces, favorisant ainsi l'actualisation de leurs capacités d'adaptation et de résilience.

Mots clés: résilience - réadaptation traumatisme craniocérébral autodétermination - priorités d'intervention.

\section{Abstract}

This article discusses how the interdisciplinary tool Client's Intervention Priorities (CIP) enhances resiliency and self-determination supporting the client in the choice of his own goals of intervention. CIP has been developed in the context of rehabilitation for people with traumatic brain injury. It is however an interdisciplinary tool, it is not specific to any specialty, clientele or clinical context, which allows different clienteles to target priorities as well as their strengths, promoting their capacity of adaptation and resilience.

Keywords: resiliency - rehabilitation traumatic brain injury - selfdetermination - intervention priorities.

\section{AUTODÉTERMINATION ET RÉSILIENCE EN RÉADAPTATION AVEC L'OUTIL PRIORITÉS D'INTERVENTION DU CLIENT (PIC)}

Eduardo Cisneros, M.A.,

Centre de réadaptation Lucie-Bruneau, Centre de recherche interdisciplinaire en réadaptation du Montréal métropolitain (CRIR), Département de psychologie, Université de Montréal.

$$
\begin{gathered}
\text { Audrey-Kristel Barbeau, } \\
\text { Centre de réadaptation Lucie-Bruneau, } \\
\text { Centre de recherche interdisciplinaire en réadaptation } \\
\text { du Montréal métropolitain (CRIR). } \\
\overline{\text { Gilles Charette, M.A., }} \\
\text { Centre de réadaptation Lucie-Bruneau. }
\end{gathered}
$$

Geneviève Léveillé, erg.,

Centre de réadaptation Lucie-Bruneau.

Michelle McKerral, Ph. D,

Centre de réadaptation Lucie-Bruneau, Centre de recherche interdisciplinaire en réadaptation du Montréal métropolitain (CRIR), Département de psychologie, Université de Montréal.

Le concept de résilience apparaît intimement lié à l'habileté d'une personne à actualiser ses capacités lorsqu'elle doit faire face à un changement, un obstacle ou une épreuve. À la suite d'un traumatisme craniocérébral (TCC), la personne subit des pertes qui affectent plusieurs dimensions de sa personne ${ }^{1}$. Elle en prendra conscience progressivement et procédera à des ajustements, s'engageant parfois dans un processus de deuil, pour pouvoir restructurer sa vie. Cette restructuration peut être facilitée par la réadaptation. Les approches de réadaptation contemporaines en déficience physique se basent sur la notion d'autodétermination pour favoriser l'implication du client et sa motivation, ainsi que celles de ses proches, dans son processus d'intervention. La notion d'autodétermination doit se concrétiser à travers des actions thérapeutiques et à l'aide d'instruments qui favorisent l'auto-observation et l'autoévaluation.

\section{L'OUTIL PRIORITÉS D'INTERVENTION DU CLIENT (PIC)}

L'équipe de réadaptation du programme TCC du Centre Lucie-Bruneau a construit un instrument clinique qui favorise l'autoobservation et l'autoévaluation du degré de fonctionnement de la personne dans la réalisation des habitudes de vie, l'outil interdisciplinaire Priorités d'intervention du client (PIC) (Charbonneau et al., 2002).

Cet outil a été créé en réponse à un besoin d'augmenter le niveau de motivation et d'implication des usagers, et de favoriser leur prise de contrôle dans le processus de réadaptation. L'outil PIC est basé sur la classification des habitudes de vie du cadre théorique du Processus de production du handicap (Fougeyrollas et al., 1998). Les habitudes de vie qui y sont définies ont été reformulées et disposées dans un ensemble de 41 cartes que la personne doit classer en fonction du degré de réalisation qu'elle perçoit dans son quotidien. Ces habitudes couvrent tant les activités quotidiennes (déplacements, tâches ménagères, etc.) que les rôles sociaux (relations interpersonnelles, responsabilités financières, etc.). La personne est alors invitée à choisir, parmi les habitudes de vie qu'elle ne réalise pas pleinement, celles qui méritent un traitement prioritaire en réadaptation. Par la suite, dans un souci de favoriser l'autodétermination, on demande au client de se prononcer sur les raisons expliquant 
le changement dans sa participation et sur les solutions qu'il entrevoit pour augmenter sa participation. À la fin, on aide l'usager à déterminer les forces (ressources personnelles et environnementales) sur lesquelles les interventions vont se concentrer.

Sur la base de ces informations, l'équipe clinique formule les objectifs individualisés de réadaptation. Le plan de réadaptation ainsi élaboré reflète donc la perception et la volonté du client, ce qui facilite sa motivation vers un changement et la réappropriation de sa vie selon ses choix. L'exercice même d'autoévaluation et d'identification de priorités constitue une pratique de l'autodétermination, de la capacité de diriger son existence selon ses propres critères. L'instrument contribue, par le fait même, à promouvoir la résilience de la personne TCC.

L'outil PIC a démontré des valeurs métrologiques très satisfaisantes avec une consistance interne de 0,89 (Alpha de Cronbach), un coefficient de fidélité testretest de 0,88 (ICC) avec un intervalle de confiance de $95 \%$ et une constance temporelle dans la sélection des priorités (comparaison entre la $1^{\text {re }}$ et la $2^{\mathrm{e}}$ administration) de 0,87 pour les activités quotidiennes et de 0,88 pour les rôles sociaux.

Les bases théoriques de l'outil PIC sont nombreuses et incluent, entre autres, l'approche écosystémique, l'approche centrée sur le client et le concept d'autodétermination. Nous établissons un lien entre ces notions et la capacité de la personne à reprendre sa vie à la suite d'une blessure cérébrale ou de toute autre condition neurologique, psychiatrique ou de santé en général.

L'approche écosystémique consiste à privilégier les interventions dans le milieu de vie de la personne de façon à faciliter l'intégration sociale. Elle se distingue des autres approches par sa façon de comprendre l'humain non pas en tant que seul élément considéré et analysé, mais en accordant également de l'importance aux différents systèmes dont l'individu fait partie (Waller, 2001; Lounsbury et al., 2009; Naaldenberg et al., 2009). Cela inclut les environnements familiaux, sociaux, culturels, scolaires ou professionnels dans lesquels évolue l'individu. Les interventions effectuées suivant l'approche écosystémique en contexte de réadaptation visent à s'intégrer à ces systèmes et à s'harmoniser à la réalité de vie de la personne. À titre d'exemple, on favorise la continuité de services, l'implication de la famille et des proches, ainsi que la collaboration avec les partenaires.

L'outil PIC permet de se concentrer sur les besoins du client, exprimés en termes d'habitudes de vie (HDV) et non pas uniquement en termes de symptômes ou de séquelles.
En ciblant les HDV, on vise les systèmes avec lesquelles la personne interagit, tout en considérant que la réadaptation en fait temporairement partie et que toute modification d'un élément entraîne des changements dans d'autres habitudes de vie. Le principe d'effet multiplicateur de l'intervention systémique invite les intervenants à travailler de façon interdisciplinaire de manière à impliquer des forces environnementales où la personne actualise ses habitudes de vie.

En travaillant de façon interdisciplinaire, on poursuit des objectifs qui vont au-delà des limites de chaque spécialité, permettant ainsi de toucher plusieurs dimensions de la vie de la personne. Par exemple, la mise en place d'un outil organisateur pour que la personne puisse gérer ces comptes a des répercussions favorables sur la gestion de l'énergie, l'amélioration des relations interpersonnelles et la confiance personnelle.

L'approche centrée sur l'usager consiste à reconnaître la capacité de l'individu à effectuer des choix et à prendre en main sa réadaptation. Les concepts dérivant de cette approche se basent sur l'autonomie individuelle, la responsabilité partagée de l'individu et de l'intervenant, la concordance contextuelle, l'accessibilité, la capacité d'action, l'aptitude à faire des choix et le respect pour la diversité (Law et al., 1995). Cette approche est fortement liée au concept d'autodétermination.

Comme décrit dans le Mémoire $d u$ Programme pour les personnes ayant subi un traumatisme craniocérébral (Direction des programmes, 2006), les conditions favorisant la mise en œuvre de cette approche en réadaptation sont les suivantes:

- fournir à la personne toutes les informations la concernant et qui pourront l'aider à faire des choix éclairés en ce qui la concerne;

- s'assurer d'être réellement à l'écoute de la personne et de respecter son rythme;

- laisser à la personne la prise de décision concernant les objectifs qu'elle juge prioritaires et les moyens privilégiés pour les atteindre;

- faire ressortir les compétences de la personne;

- assurer la transparence dans la relation usager / intervenant.

L'outil PIC permet l'actualisation de ces notions. C'est un instrument centré sur l'usager en ce sens où il fait appel à la capacité de l'individu à prendre ses propres décisions et où il engage les intervenants à prendre en compte ses choix dans le processus de réadaptation. La responsabilité de l'atteinte des buts est partagée, car client comme intervenants s'engagent dans la démarche du plan d'intervention.
Par expérience, les intervenants ont observé que les habitudes de vie jugées prioritaires par l'usager ne sont pas nécessairement celles qui sont le plus perturbées selon l'opinion de l'usager lui-même et des intervenants. Ce fait suggère que l'acte volitif de choisir est prédominant lors de l'administration du PIC, c'est-à-dire que les réponses du client sont le produit du libre arbitre et de la capacité individuelle de choisir ce qui est prioritaire. De plus, le fait de se montrer à l'écoute, par le biais de l'outil, et de traduire les préoccupations de l'usager en objectifs de réadaptation favorise l'implication et la motivation de celui-ci envers sa réadaptation.

Le concept d'autodétermination renvoie à l'habileté de l'individu de décider des choses qui le concernent, à sa capacité de préciser ses besoins, ses objectifs et son rôle dans le processus de réadaptation. Il s'agit d'un processus par lequel une personne acquiert la maîtrise des moyens lui permettant de se conscientiser, dans une perspective de développement et d'amélioration de son environnement et de ses conditions de vie. C'est l'appropriation optimale du processus décisionnel par la personne en réadaptation et son entourage.

Pour y parvenir, les intervenants sont amenés à soutenir la personne dans la prise de conscience de ses propres forces, de ses faiblesses et de celles de son environnement, à lui offrir les ressources pour accroître son savoir et à l'aider à développer ses habiletés, dans le but d'améliorer sa participation et d'actualiser son potentiel (Falk-Rafael, 2001). L'usager est donc perçu comme l'expert de sa propre condition, puisqu'il est le plus apte à décrire subjectivement sa situation et ce qu'il expérimente au quotidien (CESAF, 1998). Cela augmente son sentiment de contrôle, lui donnant ainsi le pouvoir de mieux gérer sa condition, ainsi que sa vie en général (Aujoulat et al., 2007).

Après un TCC, la perception de soi peut être perturbée. L'incapacité de percevoir ses propres difficultés et séquelles - anosognosie - peut se présenter à des degrés divers de sévérité. Le PIC place la personne en situation d'autoobservation et d'autoévaluation même si une telle capacité peut être atteinte. Cette procédure vise l'exercice de la capacité d'autoévaluation elle-même ainsi que la capacité d'autodétermination dont le client est capable à un moment donné. Ainsi, le plan de réadaptation tient compte de la conscience de la personne à un moment donné respectant le rythme de sa récupération.

Le concept d'autodétermination a été choisi comme premier principe directeur durant la création de l'outil PIC. L'outil traduit donc la préoccupation des auteurs de donner la parole à l'usager pour favo- 
riser le partage et la communication entre usager et équipe traitante et ainsi actualiser le processus d'autodétermination de la personne. L'outil crée également des conditions propices à la réflexion sur soi, incitant à l'autoperception et au jugement de l'individu sur son fonctionnement quotidien. Finalement, l'outil PIC contribue à l'augmentation de la motivation chez l'usager puisqu'il le situe au centre de son propre processus de réadaptation en vertu de l'exercice de la capacité à faire des choix, même suite à un TCC. Le fait que le client évalue ses propres HDV favorise en fait l'utilisation d'un langage commun entre l'usager, les proches et les intervenants, évitant ainsi la distance provoquée par le jargon propre à chaque discipline.

\section{DE L'AUTODÉTERMINATION À LA RÉSILIENCE}

De récents changements de paradigmes au sein des domaines de la psychologie et de la psychiatrie ont déplacé l'attention portée à l'étude des problèmes au profit d'un intérêt à l'égard de l'étude de la santé positive (Richardson et al., 1990; Aujoulat et al., 2007), portant ainsi l'accent sur le développement du sentiment de compétence chez l'individu.

Par conséquent, on voit émerger plusieurs concepts reliés à la compétence, dont parmi eux celui de résilience. Ce dernier s'est construit au sein des domaines de la psychopathologie et des études sur le développement de l'enfant, pour expliquer comment certains individus parviennent à maintenir un style de vie sain et à devenir plus outillés lorsqu'ils font face à l'adversité, à des éléments de stress ou à des changements de vie (Richardson et al., 1990).

La résilience est donc ce processus de gestion d'événements stressants, perturbateurs et éprouvants qui permet aux individus de développer des habiletés de gestion et de protection additionnelles par rapport aux caractéristiques qui leur étaient inhérentes avant l'événement. Elle sous-entend que les processus de gestion des perturbateurs légers et sévères sont des opportunités de croissance et de développement (Richardson et al., 1990). À travers les défis, les nouvelles expériences, les stress majeurs liés aux événements de la vie, les individus utilisent des habiletés de gestion et de réintégration qui leur permettent d'apprendre, de développer de nouvelles compétences et de gérer efficacement les événements de la vie.

La notion de résilience au sein des disciplines psychopathologiques fait, de nos jours, une incursion positive dans le domaine de la réadaptation en général et de la neuro-réadaptation en particulier. Grâce à des auteurs comme Cyrulnick
(2002) et Csikszentmihalyi (2004), le développement et la transmission de cette notion fournissent de solides bases aux chercheurs tels que Bonanno et ses collègues (Bonnano et al., 2002; Bonnano, 2004, 2009; Bonnano et al., 2005, Bonnano et al., 2007) pour le développement de la recherche et la création de stratégies et de cadres conceptuels favorisant la croissance personnelle.

L'opérationnalisation de ces concepts visant la traduction en gestes cliniques concrets suscitant des changements chez les personnes atteintes d'une blessure (cérébrale ou autre) a passé notamment par l'empathie, par la compréhension de la situation du patient et de sa famille, par des interventions favorisant l'autodétermination et par des suivis cliniques d'ordre psychothérapeutique. Ces interventions et cette conceptualisation se situent, au premier regard, en dehors des neurosciences et apparaissent comme des emprunts faits par la réadaptation à la psychiatrie, à la psychologie humaniste et aux disciplines reliées à la relation d'aide.

La résilience n'est pas uniquement un potentiel acquis tout au long de la vie de la personne qui fait face (et sort gagnante) de l'adversité, même si cette composante nous semble, de prime abord, nécessaire. La résilience a besoin d'un système qui interagit avec la personne, qui lui permet de développer sa capacité à «rebondir grandie » et à se réorganiser sur plusieurs plans (neurophysiologique, psychologique, relationnel) pour effectuer des tâches complexes associées au processus d'individuation. Pour ce faire, les éléments essentiels à l'apprentissage de la résilience sont la capacité d'organisation, l'estime de soi, le sentiment de compétence et la façon de réagir à des évènements catégorisés comme étant des points tournants dans la vie (Richardson et al., 1990).

Comme les recherches sur la résilience ont débuté dans les domaines de la psychiatrie et de la psychologie développementale, elles se sont centrées sur l'étude des facteurs personnels internes plutôt que sur le contexte écosystémique de l'adaptation. Toutefois, le développement du concept implique le fait que la résilience est multidimensionnelle et multidéterminée, et qu'elle est définie comme étant le produit des opérations à l'intérieur de chaque système et entre les divers niveaux systémiques à travers le temps (Waller, 2001). Le client et le programme de réadaptation constituent deux systèmes en interaction, le deuxième cherchant à favoriser le potentiel de résilience du premier.

La résilience, qu'on la perçoive comme un processus uniquement psychologique ou comme l'expression des capacités cérébrales de réorganisation fonctionnelle, requiert des actions systématiques pour la stimuler et pour la réadapter. Cette systématisation peut se nommer réadaptation, processus psychothérapeutique ou intervention psychosociale, selon la perspective clinique à partir de laquelle on la regarde, mais peu importe le nom utilisé, il doit exister un processus impliquant nécessairement la personne ellemême, son cerveau et son entourage. Dans cette dynamique, la personne ne peut rester passive: elle doit construire sa propre amélioration. La résilience ne se fait pas automatiquement, elle implique la participation active de la personne. Elle ne peut être imposée de l'extérieur, sans implication de la part de l'individu. En même temps, elle n'est pas un attribut fixe (Richardson et al., 1990), en ce sens où elle ne fait pas nécessairement partie intégrante des caractéristiques intrinsèques de l'individu: le cerveau doit avoir la capacité d'intégrer minimalement l'influence extérieure et de l'utiliser dans sa propre réorganisation. Cela suppose donc que l'intervenant peut exercer un rôle dans le développement des capacités de résilience chez l'adulte en lui fournissant les outils nécessaires à son apprentissage. De cette façon, chaque obstacle rencontré devient une opportunité de grandir, d'évoluer, de se développer et d'acquérir de nouvelles habiletés (Richardson et al., 1990). Autrement dit, les intervenants ne peuvent pas imposer leur point de vue à la personne, particulièrement quand celle-ci se rend à la phase de réintégration socioprofessionnelle et communautaire. Pour que la personne puisse avoir la possibilité de s'autodéterminer, l'équipe de réadaptation doit faciliter l'exercice de l'autodétermination. Sans l'autodétermination et une motivation intrinsèque, la résilience sera difficilement développée à la suite d'une atteinte cérébrale.

De notre point de vue, l'outil PIC est l'un des instruments disponibles aux intervenants pour influencer favorablement l'actualisation des capacités de résilience.

\section{UTILISATION DE L'OUTIL PIC PAR UNE ÉQUIPE DE RÉADAPTATION}

Dans un contexte de réadaptation en continuelle évolution, le PIC est un instrument d'autoévaluation qui concrétise une approche axée sur l'usager, sur le respect de son individualité et de son intimité. C'est un outil qui traduit la préoccupation de ses créateurs de donner la parole à l'usager pour pouvoir la transmettre aux équipes traitantes et ainsi favoriser le processus d'autodétermination de la personne. Cet instrument peut encourager la motivation de l'usager puisqu'il le situe au centre de son propre processus de réadaptation. Il favorise de plus l'interdisciplinarité par l'utilisation d'un langage commun permettant la communication entre l'usa- 
ger, ses proches et l'équipe. Finalement, cet outil crée des conditions propices à une réflexion sur soi, incitant à l'autoperception et au développement du jugement de la personne.

Au programme TCC du Centre de réadaptation Lucie-Bruneau (réadaptation socioprofessionnelle), l'outil PIC est administré au cours des premières rencontres avec l'usager. De cette façon, on permet à l'usager d'avoir une vision d'ensemble de sa situation et on lui évite la répétition d'information aux différents intervenants. Ceux-ci peuvent donc se centrer dès le départ sur des particularités disciplinaires. Étant un outil interdisciplinaire axé sur le client, le PIC peut être administré par tous les intervenants. L'attitude suggérée à l'administrateur se veut neutre et à l'écoute afin de laisser le maximum d'espace à l'usager.

Dans sa forme actuelle, l'outil peut être utilisé avec différentes clientèles en réadaptation et avec des clientèles qui nécessitent des soins à moyen et long terme (centre d'hébergement, centre d'aide aux toxicomanes, etc.). L'outil peut également convenir à toutes clientèles présentant des déficits cognitifs : les énoncés sont rédigés de façon claire et concise, minimisant ainsi la demande cognitive; différentes pauses sont prévues durant la procédure d'administration, afin d'éviter les effets de la fatigabilité; et le mode de passation est facile (manipulation de cartes), ce qui aide les gens ayant peu de disponibilités et des difficultés de mémoire. L'expérience clinique suggère également que l'outil PIC peut être appliqué à d'autres types de clientèle, notamment les gens en perte d'autonomie en raison de maladies liées au vieillissement, de maladies neurodégénératives ou de maladies psychiatriques.

L'aptitude à surmonter des épreuves et des pertes est la résultante de plusieurs facteurs personnels (capacités d'organisation, estime de soi, etc.), ainsi que de l'interaction entre l'individu et son milieu. Après un traumatisme craniocérébral, la capacité même de «rebondir» peut être perturbée par la blessure cérébrale. Les séquelles cognitives et émotionnelles ainsi que la possibilité de recevoir un soutien systématique de l'environnement influeront sur la récupération de la capacité d'autodétermination de la personne.

La réadaptation, en tant que processus systématique visant l'instauration de stratégies et d'attitudes favorisant la récupération de la personne et la réintégration socioprofessionnelle et communautaire, vient jouer un rôle essentiel dans le processus de résilience de la personne.

Parmi les stratégies favorisant la résilience de la personne ayant subi un traumatisme craniocérébral, l'outil Priorités d'intervention du client (PIC) peut jouer un rôle significatif en permettant à la personne une réflexion sur soi et sur ses capacités, mais particulièrement en offrant au patient le choix d'intégrer ses propres priorités à son processus de réadaptation. Prendre la décision de choisir les habitudes de vie qui vont intégrer son plan d'intervention est un important moyen pour susciter la motivation intrinsèque et l'implication de l'individu dans son programme de réadaptation. L'actualisation du potentiel d'autodétermination est un processus complexe qui se concrétise par la pratique même de l'autodétermination, sans que la méconnaissance des déficits ou des autres séquelles puissent en constituer un obstacle majeur. Par la facilitation de l'autodétermination à travers la liberté de choisir les habitudes de vie qui constitueront les objectifs de traitement, le PIC contribue à la reprise du pouvoir sur soi et sur sa vie, éléments associés à la capacité de réorganiser sa vie, sur de nouvelles bases et de nouvelles forces. Ainsi, l'instrument peut contribuer à la résilience de certains individus ayant subi une blessure cérébrale ou d'autres maladies physiques, des maladies liées au vieillissement, de troubles psychiatriques ou de maladies dégénératives.

\section{Bibliographie}

AUJOUlAT, I., W. D'HOORE et A. DECCACHE (2007). "Patient empowerment in theory and practice: Polysemy or cacophony? ", Patient Education and Counseling, vol. 66 , p. 13-20.

BONANNO, G.A. (2004). "Loss, trauma, and human resilience», American Psychologist, $\mathrm{n}^{\circ} 59$, p. 20-28.

BONANNO, G.A. (2009). The Other Side of Sadness: What the New Science of Bereavement Tells Us About Life After Loss, New York, Basic Books.

BONANNO, G.A., S. GALEA, A. BUCCIARELLI et D. VLAHOV (2007). "What predicts psychological resilience after disaster? The role of demographics, resources, and life stress ", Journal of Consulting and Clinical Psychology, $\mathrm{n}^{0} 75$, p. 671-682.

BONANNO, G.A., J.T. MOSKOWITZ, A. PAPA et S. FOLKMAN (2005). "Resilience to loss in bereaved spouses, bereaved parents, and bereaved gay men ", Journal of Personality and Social Psychology, $\mathrm{n}^{\circ} 88$, p. 827-843.

BONANNO, G.A., C.B. WORTMAN, D.R. LEHMAN, R.G. TWEED, M. HARING, J. SONNEGA, D. CARR et R.M. NESSE (2002). «Resilience to loss and chronic grief: A prospective study from preloss to 18-months postloss ", Journal of Personality and Social Psychology, $\mathrm{n}^{\circ}$ 83, p. 1150-1164.

CENTRE D'EXCELLENCE POUR LA SANTÉ DES FEMMES - CONSORTIUM UNIVERSITÉ DE MONTRÉAL (CESAF) (1998). «Empowerment», en ligne, <http:// www.cesaf.umontreal.ca/f.ress.doss.empow. doc1.html>, consulté le 2010-08-09.
CHARBONNEAU, A., G. CHARRETTE, E. CISNEROS, J.-P. DROUIN, É. LEMAY, G. LÉVEILLÉ et M. MCKERRAL (2002). L'outil Priorités d'intervention du client (PIC), Programme pour des personnes ayant subi un traumatisme craniocérébral, Centre de réadaptation Lucie-Bruneau, Montréal.

CSIKSZENTMIHALYI, M. (2004). Vivre: psychologie du bonheur, Paris, Robert Lafont.

CYRULNIK, B. (2002). Un merveilleux malheur, 2 e édition, Paris, Odile Jacob.

DIRECTION DES PROGRAMMES CLIENTÈLES (2006). Mémoire du Programme pour de personnes ayant subi un traumatisme craniocérébral, Centre de réadaptation LucieBruneau, Montréal.

FALK-RAFAEL, A.R. (2001). "Empowerment as a process of evolving consciousness: A model of empowered caring ", Advances in Nursing Science, vol. 24, ${ }^{\circ}$ 1, p. 1-16.

FOUGEYROLLAS, P., R. CLOUTIER, H. BERGERON, J. COTÉ et G. ST-MICHEL (1998). Classification québécoise du Processus de production du handicap, Réseau international sur le Processus de production du handicap, Québec.

LAW, M., S. BAPTISTE et J. MILLS (1995). "Client-centered practice: What does it mean and does it make a difference?», Canadian Journal of Occupational Therapists, vol. 62, $\mathrm{n}^{\circ} 5$, p. $250-257$.

LOUNSBURY, D.M. et S.G. MITCHELL (2009). "Introduction to special issue on social ecological approaches to community health research and action", American Journal of Community Psychology, vol. 44, $\mathrm{n}^{\text {os }}$ 3-4, suppl. 2, p. 13-20.

NAALDENBERG J., L. VAANDRAGER, M. KOELEN, A.M. WAGEMAKERS, H. SAAN et K. DE HOOG (2009). "Elaborating on systems thinking in health promotion », Global Health Promotion, vol. 16, $\mathrm{n}^{\circ}$ 1, p. 39-47.

RICHARDSON, G.E., B.L. NEIGER, S. JEMSENS et K.L. KUMPLER (1990). «The resiliency model », Health Education, vol. 21, p. 33-39.

WALLER, M.A. (2001). "Resilience in ecosystemic context: Evolution of the concept », American Journal of Orthopsychiatry, vol. 71, p. 290-296.

\section{Note}

1. Un TCC survient à la suite d'un impact à la tête suffisamment intense pour créer des blessures au cerveau. Contrairement aux autres types de tissus humains qui, lorsque abimés, se régénèrent, une blessure du système nerveux central n'est pas suivie d'une reproduction cellulaire. Les neurones, responsables de la transmission nerveuse et d'une partie des processus psychologiques qu'elle soutient, meurent. Le cerveau réagit à la mort cellulaire en entamant un processus de réorganisation qui, même partiel, permet à la personne de s'ajuster aux changements provoqués par la mort des cellules. Toutefois, la récupération ne dépend pas uniquement d'une capacité neurobiologique à se réorganiser, elle est aussi influencée par le niveau de motivation et de participation de la personne ayant subi la blessure. 